\title{
Tackling osteoporosis and fragility fractures in Singapore
}

\author{
Manju Chandran, ${ }^{1,2,3,4}$ MD, Paul J Mitchell, ${ }^{3,5,6,7}{ }_{B S C}$
}

A shift in worldwide population ageing demographics has occurred in the 21 st century. The longevity miracle is most keenly felt in Singapore, a young nation which gained its independence only in 1965, but has one of the fastest growing ageing populations in the world. It is estimated that by 2030, 1 in 4 people in Singapore will be aged over 65 years. This will rise to almost 1 in 2 by $2050 .{ }^{1}$ Given the multitude of documented positive contributions the elderly can bring to society, this should be viewed positively and not as problematic. However, one cannot ignore the stark reality that this steep increase in the old-age dependency ratio (ratio of population aged 65 and over, to that aged 15-64 years), which was 21.3 in 2020 and is predicted to increase to 60.6 by 2050 in Singapore, ${ }^{2}$ will bring with it a dramatic increase in the incidence of age-related chronic non-communicable diseases in its wake.

Osteoporosis, a systemic skeletal disease characterised by low bone mass and microarchitectural deterioration of bone tissue, with a consequent increase in bone fragility and susceptibility to fracture, ${ }^{3}$ imposes a huge health-economic burden on societies and individuals. The number of women who will experience a fracture annually exceeds the combined number of women who will experience incident breast cancer, myocardial infarction, or stroke across all ethnic groups. ${ }^{4}$ In the status quo scenario if no urgent interventions are made, it is projected that the total number of osteoporotic fractures in Singapore will increase from 15,267 in 2017 to 24,104 in 2035.5 This represents a $57.9 \%$ rise in less than 20 years. The overarching costs that this phenomenal increase in fractures in Singapore would incur is in the millions, with total costs predicted to be SGD289.6 million in 2035. However, despite this knowledge and an abundance of data demonstrating the impact of fractures on individual health (such as reduced physical function, impaired mobility, loss of independence, and increased risk of premature death) associated with osteoporosis,,${ }^{6,7}$ a very real existential crisis is prevalent with a ubiquitous and universal care gap seen in Singapore as in the rest of the world. In a study conducted a mere 7 years ago, it was found only $28 \%$ of first-time hip fracture patients in Singapore received prescription medications for osteoporosis in the 6 months after discharge. ${ }^{8}$

Singapore is one of the few countries in the Asia Pacific where osteoporosis is considered a national health priority. It also boasts Asia's oldest Fracture Liaison Service-Osteoporosis Patient Targeted and Integrated Management for Active Living (OPTIMAL). Since its inception in the public hospitals in 2008, OPTIMAL has helped to increase rates of referral for Dual Energy X-Ray Absorptiometry scans, for diagnosis and treatment of osteoporosis, and compliance rates to osteoporosis medications compared to worldwide figures. ${ }^{9}$ OPTIMAL 2.0, launched in 2019, has been incorporated into the operational budget of public restructured hospitals and polyclinics in Singapore. This move aims to make secondary fracture prevention a part of the routine workflow of these centres.

Following successful lobbying by healthcare providers, osteoporosis was also included in the Chronic Disease Management Programme in 2015, whereby patients can use Medisave, the compulsory national health insurance in Singapore, to pay for outpatient clinic visits pertaining to their osteoporosis care.

Stellar work in increasing awareness about osteoporosis among the lay public and healthcare professionals is being done by the Osteoporosis Society Singapore and by individual units such as the Osteoporosis and Bone Metabolism Unit at Singapore General Hospital. However, single agencies cannot successfully achieve integrated service delivery in a consistent and reliable way, unless they work in partnership. Alliances such as the Bone Alliance Singapore formed in 2018 that bring

\footnotetext{
${ }^{1}$ Osteoporosis and Bone Metabolism Unit, Department of Endocrinology, Singapore General Hospital, Singapore

${ }^{2}$ Duke-NUS Medical School, Singapore

${ }^{3}$ Asia Pacific Consortium on Osteoporosis (APCO)

${ }^{4}$ International Osteoporosis Foundation

${ }^{5}$ School of Medicine, University of Notre Dame, Australia

${ }^{6}$ Nuffield Department of Orthopaedics, Rheumatology and Musculoskeletal Sciences, University of Oxford, United Kingdom

${ }^{7}$ Osteoporosis New Zealand

Correspondence: A/Prof Manju Chandran, Osteoporosis and Bone Metabolism Unit, Department of Endocrinology, Singapore General Hospital, Academia Building, 20 College Road, Singapore 169856.

Email: manju.chandran@singhealth.com.sg
} 
together healthcare professionals, academics, government agencies, voluntary welfare organisations and patient groups, hold great promise in addressing the gaps in bone health in a comprehensive, action-oriented way. Such multistakeholder involvement will ensure that policies and initiatives all point in the same direction. This approach also ensures that management will not only include medical and surgical treatment of osteoporosis and fractures, but also community interventions such as initiatives that encourage exercise and healthy lifestyle habits and prevent falls.

Osteoporosis medical therapy has slowly moved from the serendipitous pharmacology that it was based on in years past, to more targeted treatment with the introduction of medications such as the monoclonal antibody against RANK ligand (Denosumab), anabolic agents such as human recombinant parathyroid hormone (Teriparatide), and the monoclonal antibody against sclerostin (Romosozumab). However, the field is still riddled with controversies, and gaps exist in available evidence to guide physicians on appropriate management of their patients. Questions remain on when screening for osteoporosis and fracture risk should begin; what screening methodology should be employed; at what threshold of fracture risk should treatment be started; and the type of fracture risk assessment methodology to be used. Opinions continue to be divided on how long treatment should be given, and whether men who are at lower risk for osteoporosis compared to women should have different screening and intervention thresholds. It is imperative that clearer and more comprehensive guidelines be developed on the appropriate management of bone loss associated with hormonal therapy for breast and prostate cancer, and that associated with the use of glucocorticoids. Research into standardising vitamin $\mathrm{D}$ and bone turnover marker assays so that they can be employed appropriately in clinical practice is necessary. Unlike other chronic diseases such as diabetes, a potential goal or target for osteoporosis treatment is unclear and still beyond our reach. We need to elucidate ways of identifying the patient at imminent fracture risk, and address the risks associated with prolonged use of antiresorptive medications such as atypical femoral fractures. A critical need exists to fine-tune recent developments in artificial intelligence that have been applied to the assessment of osteoporosis and the modelling of fracture risk. Candidate gene and genome-wide association studies that could potentially open up pharmacogenomic pathways for osteoporosis treatments must be explored.

Although high-quality research is being conducted by some individuals and centres in Singapore on topics as varied as secondary osteoporosis, ${ }^{10}$ cost-effectiveness of medical therapies and intervention thresholds for osteoporosis, ${ }^{11}$ epidemiology of fractures, ${ }^{12}$ etc., competing goals and fragmentation of work into silos are evident in the country. Despite investment in infrastructure for health research in chronic conditions such as diabetes having increased tremendously over the past quinquennium, research on osteoporosis and on rare skeletal diseases that are associated with bone loss remains poorly funded and appreciated. Debates on funding models, and the appropriate balance between research driven by investigators for specific problems and that driven by policy, should be initiated, as they are imperative to drive meaningful research agendas. Ensuring partnership between scientists, clinicians, stakeholders from science and technology, education, health-related areas in the public and private sectors and governmental agencies is necessary to foster a conducive environment for musculoskeletal research, and to put ivory tower ideas into action. Finally, involving patients and the public in research agendas so that they are of relevance to them, and important topics that scientists and clinicians may not have previously considered can be identified, is critical to provide in-depth understanding of priorities for people living with, or caring for those with the devastating condition of osteoporosis.

Even though the aforementioned are steps forward in the right direction, Singapore cannot afford to remain in a vacuum and should join forces with extraneous entities and countries to tackle the huge burden of osteoporosis in the Asia Pacific. Collaborating with organisations such as the International Osteoporosis Foundation (www.osteoporosis.foundation) and the newly formed Asia Pacific Consortium on Osteoporosis (APCO, www.apcobonehealth.org) will facilitate this.

APCO was launched in May 2019 with the vision of reducing the burden of osteoporosis and its complication of fragility fractures in the region. It comprises 39 osteoporosis experts from 19 countries and territories, including Singapore. These experts from both public and private healthcare systems cover the clinical spectrum of specialties that manage osteoporosis. The guiding principle of APCO rests on fostering harmonisation of osteoporosis care across the region. The consortium has developed a pan-Asia Pacific Framework of Clinical Standards of Care through a comparative analysis of the extant guidelines in the region and using the Delphi process of consensus derivation. ${ }^{13}$ The framework consists of a set of clear, concise, relevant, and pragmatic clinical standards that can be adapted to meet individual national requirements 
and can serve as a benchmark for Singapore to revise its own Appropriate Care Guide ${ }^{14}$ for osteoporosis produced in 2018. Countries such as New Zealand and Pakistan are considering revising their national guidelines based on the APCO Framework. One of Osteoporosis New Zealand's key activities in 2021 is to update Guidance on the Diagnosis and Management of Osteoporosis in New Zealand published in 2017.

With scientific ingenuity, critical research, pooling of local clinical resources, and productive regional and international collaborations, we can fly into the eye of the storm that is osteoporosis and tame this devastating disease.

\section{REFERENCES}

1. Prime Minister's Office Singapore. Population White Paper: A Sustainable Population for a Dynamic Singapore, 9 January 2013. Available at: https://www.strategygroup.gov.sg/media-centre/ population-white-paper-a-sustainable-population-for-a-dynamicsingapore. Accessed on 23 March 2021.

2. United Nations Department of Economic and Social Affairs Population Division.World population ageing 2019: Highlights. United Nations, New York, 2019.

3. Kanis JA, Melton LJ III, Christiansen C, et al. The diagnosis of osteoporosis. J Bone Miner Res 1994;9:1137-41.

4. Cauley JA, Wampler NS, Barnhart JM, et al. Incidence of fractures compared to cardiovascular disease and breast cancer: the Women's Health Initiative Observational Study. Osteoporos Int 2008;19:1717-23.

5. Chandran M, Lau TC, Gagnon-Arpin I, et al. The health and economic burden of osteoporotic fractures in Singapore and the potential impact of increasing treatment rates through more pharmacological options. Arch Osteoporos 2019;14:114.
6. Morin S, Lix LM, Azimaee M, et al. Institutionalization following incident non-traumatic fractures in community-dwelling men and women. Osteoporos Int 2012;23:238-6.

7. Morin S, Lix LM, Azimaee M, et al. Mortality rates after incident non-traumatic fractures in older men and women. Osteoporos Int 2011;22:2239-48.

8. SP, Lee JK, Koh L, Soong YK, et al. Factors influencing diagnosis and treatment of osteoporosis after a fragility fracture among postmenopausal women in Asian countries: a retrospective study. BMC Womens Health 2013;13:7.

9. Chandran M, Tan MZ, Cheen M, et al. Secondary prevention of osteoporotic fractures-an "OPTIMAL" model of care from Singapore. Osteoporos Int 2013;24:2809-17.

10. Yung CK, Fook-Chong S, Chandran M. The prevalence of recognized contributors to secondary osteoporosis in South East Asian men and post-menopausal women. Are Z score diagnostic thresholds useful predictors of their presence? Arch Osteoporos 2012;7:49-56.

11. Chandran M, Ganesan G, Tan KB, et al. Cost-effectiveness of FRAX ${ }^{\circledR}$-based intervention thresholds for management of osteoporosis in Singaporean women. Osteoporos Int 2021; 32:133-44.

12. Yong EL, Ganesan G, Kramer MS, et al. Hip fractures in Singapore: ethnic differences and temporal trends in the new millennium. Osteoporos Int 2019;30:879-86.

13. Chandran M, Mitchell PJ, Amphansap T, et al. Asia Pacific Consortium on Osteoporosis (APCO). Development of the Asia Pacific Consortium on Osteoporosis (APCO) Framework: clinical standards of care for the screening, diagnosis, and management of osteoporosis in the Asia-Pacific region. Osteoporos Int 2021. doi: 10.1007/s00198-020-05742-0.

14. Agency for Care Effectiveness. Osteoporosis - identification and management in primary care, 7 November 2018. Available at: https:// www.ace-hta.gov.sg/healthcare-professionals/ace-clinical-guidances(acgs)/details/osteoporosis-identification-and-management-in-primarycare. Accessed on 23 March 2021. 